\title{
Changes of Antioxidant Capacity of Buckwheat Extracts during Germination
}

\author{
Zhang Guotao \\ Yulin College, Yulin City 719000, Shaanxi Province, China \\ 994527145@qq.com
}

\begin{abstract}
Keywords: buckwheat; antioxidant; germination
Abstract. In order to find buckwheat having higher antioxidant compounds and antioxidant ability. In this study, we evaluated total phenolic content, DPPH radical scavenging rate, ABTS radical scavenging rate, reducing activity and Fe2+ chelation activity of buckwheat extracts after and before germination, and during germination. At different germination time, we found significant differences of total phenolic content and antioxidant ability. With germination time being prolonged, total phenolic content increased and antioxidant ability became stronger, but this trends became weak after germination for 5 days.
\end{abstract}

\section{Introduction}

The pathogenesis of various disorders and diseases was related to oxidative stress. In order to having healthy life, we can absorb the content of antioxidants in a diet, which prevented many disease occurred. Therefore, many of scientists start to be interested in how to find materials with rich antioxidants.

Buckwheat is one of the ancient cereal crops. In the past, buckwheat was mainly used for food purposes. But now, due to having high content of biologically active compounds, buckwheat start to become materials produced functional food ${ }^{[1]}$.

Germination of seeds involves several metabolic activites. From nutritional perspective, germination increases some nutritional compounds, such as antioxidants, vitamin contents and so on, and reduces anti-nutritional factors ${ }^{[2]}$. The changes depend on germination conditions such as soaking time, soaking temperature, germination time, germination temperature, and germination humidity ${ }^{[3]}$.

The objectives of this study were to investigate the effect of germination of buckwheat on production of antioxidant compounds and antioxidant activity to assess whether germination can produce functional compounds in buckwheat.

\section{Materials and methods}

\subsection{Materials}

Fresh samples of buckwheat was purchased from the local market. Chemicals and reagents used such as DPPH, ABTS, and ferrozine were procured from Sigma company. The other chemicals and reagents was analytical reagent, and was made in China.

\subsection{Germination process of buckwheat}

Selection of buckwheat seeds $\rightarrow$ Cleaning of buckwheat seeds $\rightarrow$ Soaking of buckwheat seeds $\rightarrow$ Germination of buckwheat seeds $\rightarrow$ Collect buckwheat seeds germinated.

\subsection{Buckwheat extracts}

Buckwheat extracts was made by ultrasonic extraction technology. The buckwheat extract was prepared by soaking $5 \mathrm{~g}$ of buckwheat flour in $100 \mathrm{~mL}, 80 \%$ methanol solution for $5 \mathrm{~min}$ in ultrasonic environment. The mixture was centrifugated and then filtered, and the filtrates was concentrated at $40{ }^{\circ} \mathrm{C}$. The flour of buckwheat extracts was made by freeze-dried method. The flour of buckwheat extract was diluted by $80 \%$ methanol, and the final concentration of extract was $2 \mathrm{mg} / \mathrm{ml}$. 


\subsection{Determination of total phenolic}

Folin-Cioocalteu reagent was used to determine total phenolic ${ }^{[4]}$. The $100 \mu \mathrm{L}$ of buckwheat extracts was mixed with two hundred microliters of Folin-Cioocalteu reagent (previously diluted onefold with distilled water) and $2 \mathrm{~mL}$ of sodium bicarbonate (10\%) solution; distilled water was added to mixture, and final volume of mixture was up to $5 \mathrm{~mL}$. After $40 \mathrm{~min}$, absorbance of the mixture was measured at $760 \mathrm{~nm}$. The concentration of total phenolic of buckwheat extracts was expressed as an equivalent of gallic acid per $1 \mathrm{~g}$ buckwheat $(\mathrm{mg} / \mathrm{g})$.

\subsection{Determination of DPPH radical scavenging capacity}

The DPPH assay was used to determinate antioxidant capacity of buckwheat extracts, and made some changes ${ }^{[5]}$. DPPH reagent was dissolved by methanol. The $100 \mu \mathrm{L}$ of buckwheat extracts was allowed to react with $3 \mathrm{~mL}$ of DPPH $(0.025 \mathrm{mg} / \mathrm{mL})$ solution for reaction time $1 \mathrm{~h}$ in no light. Absorbance of mixture was measured at $515 \mathrm{~nm}$. A control sample with no added extract also was analyzed. The following equation was used to calculate DPPH radical scavenging percentage.

$\mathrm{DPPH}$ scavenging rate $(\%)=\left[\left(\mathrm{A}_{\text {control }}-\mathrm{A}\right.\right.$ sample $\left.) /\left(\mathrm{A}_{\text {control }}\right)\right] \times 100$

A: absorbance at $515 \mathrm{~nm}$

\subsection{Determination of ABTS radical scavenging capacity ${ }^{[6]}$}

A solution of ABTS cation-radical was prepared using the reaction mixture of aqueous solution of ABTS and K2S2O8 solution, and the final concentration of ABTS and $\mathrm{K} 2 \mathrm{~S} 2 \mathrm{O} 8$ were $7 \mathrm{~m} \mathrm{~mol} / \mathrm{L}$ and $2.45 \mathrm{~m} \mathrm{~mol} / \mathrm{L}$, respectively. The stock solution of ABTS radical was obtained after the reaction time for 12 to $16 \mathrm{~h}$ under the laboratory temperature at darkness. The final working solution was obtained by the stock solution diluted by distilled water, and was to the absorbance of $0.6 \pm 0.02$ at $734 \mathrm{~nm}$. The $100 \mu \mathrm{L}$ of buckwheat extracts and $3 \mathrm{~mL}$ ABTS working solution were mixt to react for 30 min under the laboratory temperature at darkness. Absorbance of reaction solution was measured at $734 \mathrm{~nm}$. A control sample with no added extracts also was analyzed. The following equation was used to calculate ABTS radical scavenging percentage.

ABTS scavenging capacity $(\%)=[($ A control - A sample $) /($ A control $)] \times 100$

A: absorbance at $734 \mathrm{~nm}$

\subsection{Reducing property}

The ability of the extract to reduce a $\mathrm{FeCl}_{3}$ solution was used to assess reducing property of the buckwheat extracts ${ }^{[7]}$. The $200 \mu \mathrm{L}$ buckwheat exracts was mixed with $1 \mathrm{~mL}, 200 \mathrm{~m}$ mol / L sodium phosphate (pH6.6) and $1 \mathrm{~mL} 1 \%$ potassium ferricyanide. The mixture was incubated at $50^{\circ} \mathrm{C}$ for 20 min. Then $1 \mathrm{~mL}, 10 \%$ TCA was added to the mixture. The final mixture was centrifuged at 4000 $\mathrm{r} / \mathrm{min}$ for $10 \mathrm{~min}$. A $2 \mathrm{~mL}$ of supernatant was mixed with an equal volume of water and $0.4 \mathrm{~mL}, 0.1 \%$ ferric chloride. The absorbance of the final mixture was taken at $700 \mathrm{~nm}$. The same treatment was performed to a standard ascorbic acid solution. The reducing power was calculated and expressed as an equivalent of ascorbic acid per $1 \mathrm{~g}$ buckwheat $(\mathrm{mg} / \mathrm{g})$.

\section{$2.8 \mathrm{Fe}^{2+}$ chelation assay}

$\mathrm{Fe}^{2+}$ chelation ability of buckwheat extracts was calculated using a modified method ${ }^{[8]}$. Methanol was added to the reaction mixture containing freshly prepared $1 \mathrm{~mol} / \mathrm{L} \mathrm{FeSO} 4(25 \mu \mathrm{L}), 200 \mu \mathrm{L}$ of buckwheat extracts, and $50 \mu \mathrm{L}, 5 \mathrm{~m} \mathrm{~mol} / \mathrm{L}$ ferrozine, and the final volume of the mixture was keep at $3 \mathrm{~mL}$. The reaction mixture was incubated for $10 \mathrm{~min}$, and absorbance was measured at $562 \mathrm{~nm}$. The $\mathrm{F}^{\mathrm{e} 2+}$ chelation ability was expressed as equivalent of EDTA-Na2 per $1 \mathrm{~g}$ buckwheat (mg/g).

\section{Results and discussion}

\subsection{Changes of total phenolic concentration during buckwheat germination}

Changes of total phenolic content of buckwheat extracts during germination is presented in Fig.1.The result showed that total polyphenol content of buckwheat extracts for non-germination was $8.4 \mathrm{mg} / \mathrm{g}$, and for germination total phenolic increased to $9.06-12.06 \mathrm{mg} / \mathrm{g}$. All germination buckwheat extracts had significantly higher concentration than non-germination buckwheat. It is obvious that with germination time increasing, total phenolic content of buckwheat extracts increased, and this trend became weak after 5 days of germination. 


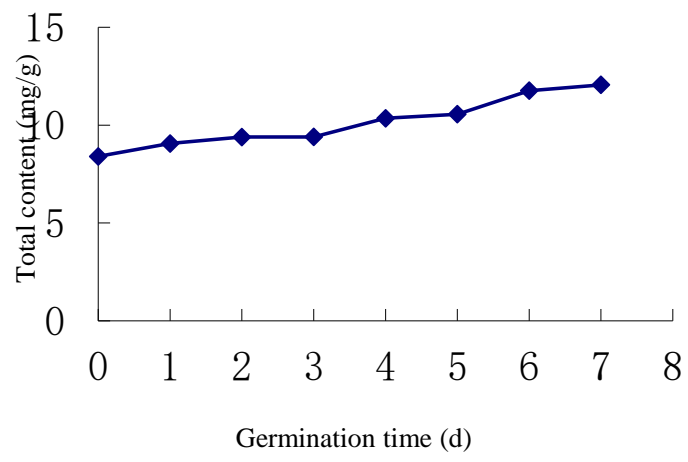

Fig.1 Changes of toal phenolic concentration during buckwheat germination

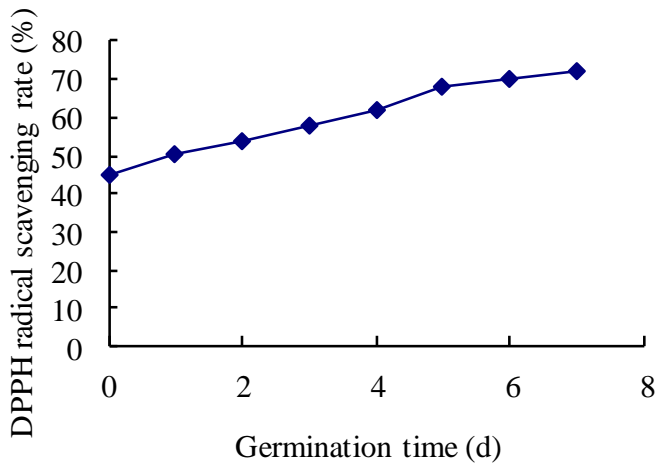

Fig.2 Changes of DPPH radical scavenging capacity during buckwheat germination

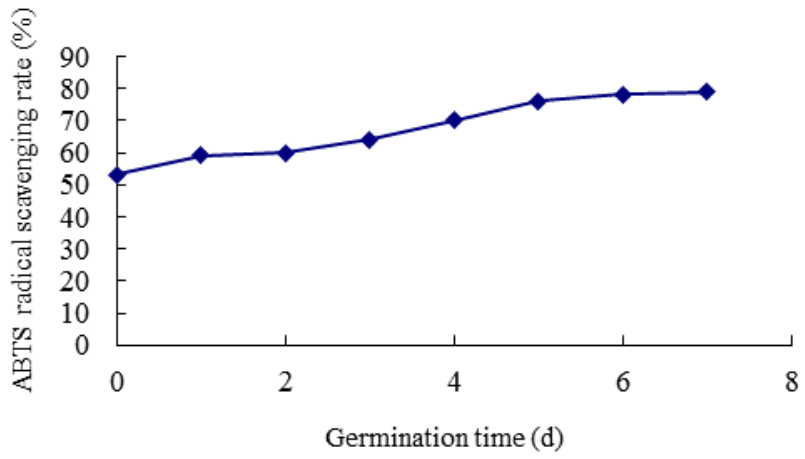

Fig. 3 Changes of ABTS radical scavenging capacity during buckwheat germination

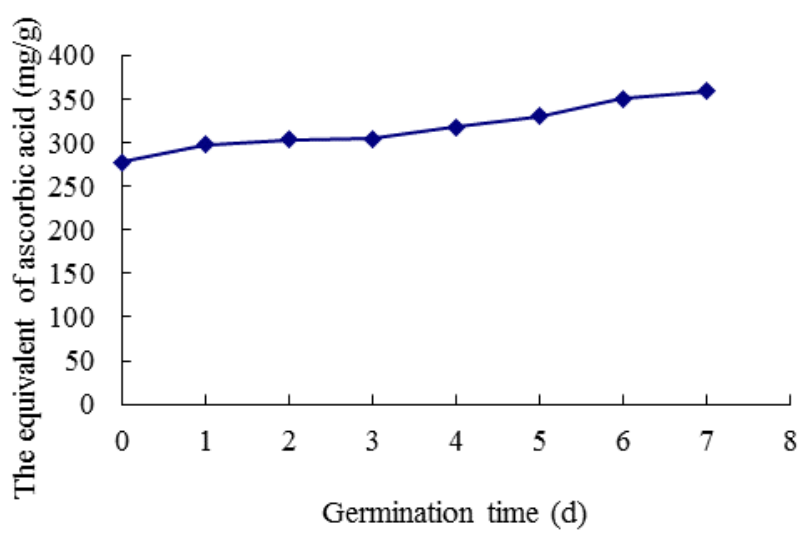

Fig.4 Changes of reducing property during buckwheat germination

\subsection{Changes of DPPH radical scavenging capacity during buckwheat germination}

DPPH radical scavenging rate increased significantly with germination time being prolonged, and this for germination buckwheat extracts was stronger than non-germination (Fig.2). DPPH radical scavenging rate revealed a similar trend to total phenolic content, and the maximum DPPH radical scavenging rate was up to $72 \%$, and higher in germination buckwheat seeds than in non-germination seeds $(45.1 \%)$. Before 5 days, changes of DPPH radical scavenging rate increased obviously. After 5 days, however, the changes of DPPH radical scavenging capacity became weak.

\subsection{Changes of ABTS radical scavenging capacity during buckwheat germination}

Fig.3 showed the changing trend of ABTS radical scavenging capacity with germination time of buckwheat seeds. It was found that ABTS radical scavenging rate increased remarkably with germination time during germination. During early germination stage, ABTS radical scavenging rate grow rapidly. But it grow slowly after 5 days. The maximum ABTS radical scavenging rate was 79\%, and the value was about 1.5 times that of non-germination buckwheat seeds.

\subsection{Changes of reducing property during buckwheat germination}

The equivalent of asorbic acid in per $1 \mathrm{~g}$ buckwheat extracts was used to evaluate reducing property of buckwheat extracts, and the higher value of the equivalent of asorbic acid, there are the stronger of reducing property of buckwheat extracts. With germination time increasing, the value of the equivalent of asorbic acid increased (Fig.4). Therefore, it can be concluded that germination was benefit to improve reducing property of buckwheat extracts.

\subsection{Changes of $\mathrm{Fe}^{+}$chelation ability during buckwheat germination}

The $\mathrm{Fe} 2^{+}$chelation ability was expressed as equivalent of EDTA-Na2 per $1 \mathrm{~g}$ buckwheat extracts. The higher value of the equivalent of EDTA-Na2, the reducing property of buckwheat extracts was 
stronger. Fig. 5 showed that the changing trend of $\mathrm{Fe}^{2+}$ chelation ability with germination time of buckwheat seeds. It was found that $\mathrm{Fe}^{2+}$ chelation ability increased with germination time during germination.

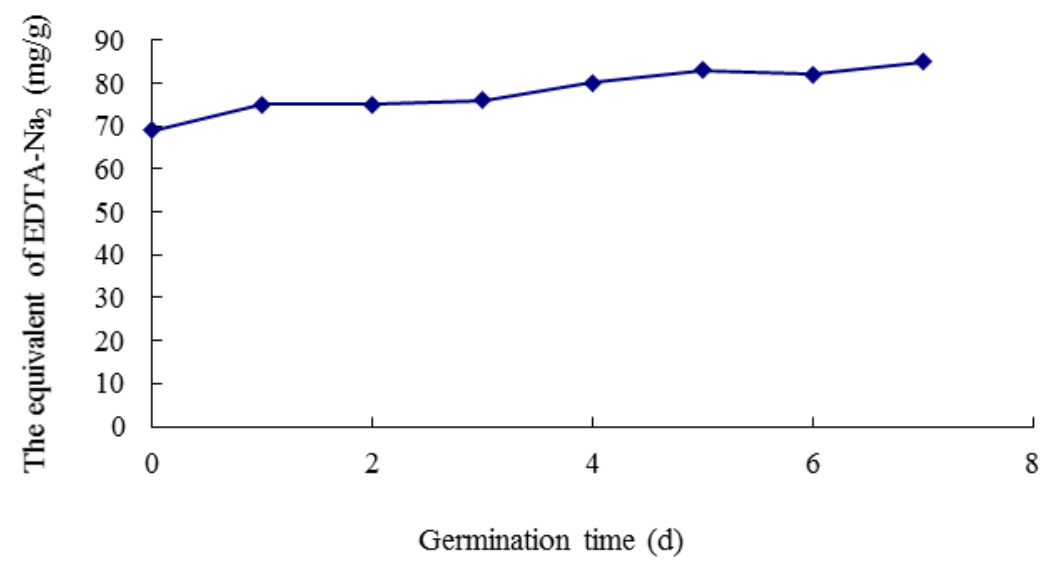

Fig. 5 Changes of $\mathrm{Fe}^{2+}$ chelation ability of buckwheat extract during germination

\section{Summary}

The total phenolic content, DPPH radical scavenging rate, ABTS radical scavenging rate, reducing activity and $\mathrm{Fe}^{2+}$ chelation ability in germinating buckwheat seeds were analyzed in this paper. Germination can improve buckwheat antioxidant compounds, and increase antioxidant ability. Moreover, this study provides the scientific basis and the effective method for making functional buckwheat foods.

\section{Acknowledgements}

This work was supported by the project of science and technology of Shaanxi Province (No. $2011 \mathrm{KW}-27$ ).

\section{References}

[1] L Wang, XD Li, MN Niu, et al: Journal of cereal science Vol. 58(2013), p.348-354.

[2] O.N Donkor, A Henriksson, T Vasiljevic, et al: Journal of food science Vol.70 (2005), p 375-381.

[3] L Lopez-Barrios, JA Gutierrez-Uribe, SO Serna-Saldivar: Journal of food science Vol.79（2014） p. 273-283.

[4] KK. Adom, RH Liu: Journal of agricultural and food chemistry Vol. 50(2002), p.6182-6187.

[5] M Hao, T Beta: Food chemistry Vol.133 (2012), p. 1320-1325.

[6] P Siddhuraju: LWT - Food Science and Technology Vol.40 (2007), p. 982-990.

[7] M Oyaizu: Journal of Nutrition Vol.44 (1986), p. 307-315.

[8] R Singh, S Singh, S Kumar, S Arora: Food and Chemical Toxicology Vol.45(2007), p.1216-1223. 